\title{
Disrupting a survival signal
}

Pancreatic adenocarcinoma (PDA) is a notoriously desmoplastic tumour type, a trait that is thought to contribute to the lack of response that this tumour type shows to chemotherapy. The use of mouse models of PDA that closely resemble the human disease has shown that disrupting or reducing the stroma surrounding the tumour cells can improve response rates, but it is not clear whether this is because of an increased concentration of chemotherapy reaching the tumour cells.

To address this, Albrecht Neesse, David Tuveson and colleagues used a mouse PDA model to investigate whether increased levels of gemcitabine within the tumour, without manipulating the tumour stroma, would improve therapeutic outcome. Phosphorylation of gemcitabine in cells generates the active form of the drug, however, gemcitabine is also rapidly degraded to an inactive form by cytidine deaminase that is present both in blood and inside cells. The group used an inhibitor of cytidine deaminase (3,4,5,6-tetrahydrouridine

understanding tumour cellstromal cell interactions might provide greater insight into drug resistance mechanisms (THU)) to increase the intratumoural and plasma levels of the active form of gemcitabine, but this did not increase the rate of apoptosis in the tumour or slow tumour growth in the mice.

Next, the authors turned their attention to the stroma and investigated the effect of targeting connective tissue growth factor (CTGF), which is abundantly expressed in both mouse and human PDA. CTGF is highly expressed by cancer-associated fibroblasts (CAFs) and is expressed at low levels by tumour cells. Inhibition of CTGF using FG-3019, an antibody against CTGF that is currently in clinical trials, had no effect on tumour growth. However, the combination of FG-3019 and gemcitabine reduced tumour burden and increased the levels of apoptosis that were evident in the tumours, compared with tumours that were treated with gemcitabine alone. Interestingly, the authors found that it was not the CAFs that were undergoing apoptosis, but the epithelial tumour cells and that the levels of gemcitabine metabolites within the tumour were not increased compared with tumours treated with gemcitabine alone. Further analyses of proteins known to regulate apoptosis showed that the expression of X-linked inhibitor of apoptosis (XIAP) protein was reduced in the tumour cells as a result of treatment with FG-3019, and whole-genome expression analysis of tumour mRNA showed that only a few mRNAs, including Xiap, Birc6 and Hifla, were substantially downregulated in tumours treated with FG-3019 and gemcitabine.

A randomized treatment study in the PDA mouse model indicated that, although the combination of FG-3019 and gemcitabine resulted in a slowing of tumour growth and an extended median survival compared with mice treated with gemcitabine alone, there were no obvious differences in the tumour histopathology between mice receiving gemcitabine and mice receiving gemcitabine with

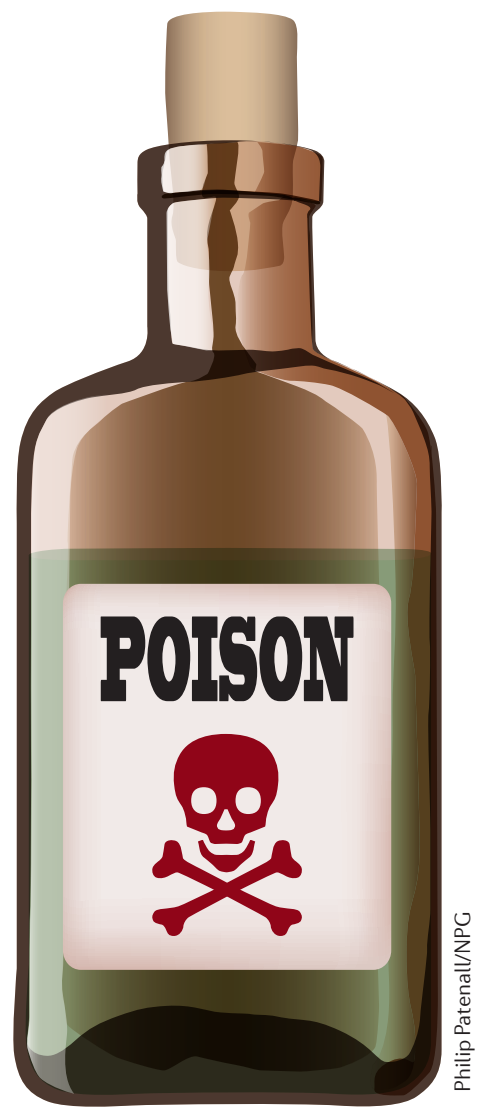

FG-3019. However, the mice receiving the combined treatment had fewer liver metastases.

The authors conclude that blocking CTGF probably interferes with a survival signal required by the epithelial tumour cells to survive in the presence of gemcitabine, and that understanding tumour cell-stromal cell interactions might provide greater insight into drug resistance mechanisms.

Nicola McCarthy

ORIGINAL RESEARCH PAPER Neesse, A. et al. CTGF antagonism with mAb FG-3019 enhances chemotherapy response without increasing drug delivery in murine ductal pancreas cancer. Proc.

Natl Acad. Sci. http://www.pnas.org/

content/110/30/12325 (2013) 